\title{
A KIND OF COORDINATED EVOLUTION MEASUREMENT MODEL FOR TRAFFIC NETWORK BASED ON COMPLEXITY DEGREE
}

\author{
Qizhou $\mathrm{HU}^{*}$, Minjia TAN \\ School of Automation, Nanjing University of Science and Technology, Nanjing, China
}

Received 14 January 2018; revised 24 October 2018, 3 February 2019; accepted 1 March 2019

\begin{abstract}
Coordinated evolution is a process with complexity, temporality, spatiality, and continuity. The existed methods cannot relevantly satisfy and measure the degree of coordinated evolution in real conditions. Aiming at solving the coordinated evolution problems for the urban traffic network, the information complexity must be evaluated, this paper uses the multi-dimensional connection number for compressing the factors of traffic network. Firstly, the basic characteristics of traffic network are analysed on the definition of traffic information complexity. The traffic network measurement model is established based on the information entropy, and the coordinated evolution process of the multi-layer urban traffic network is analysed for defining the ordered parameters of the traffic network. Then the coordinated measurement model for the multi-layer traffic network is constructed by the ordered parameters. In addition, we set up a coordinated evolution model according to the proposed estimation criteria of the ordered parameters and the theory of the multi-dimensional connection numbers. The case analysis shows that the order degree of Hangzhou traffic network is 0.7929 , which approaches to 1 as while the comprehensive coordinated index of Hangzhou multi-layer traffic network is 0.3323 , which clearly and intuitively gives a measurement value for the multi-layer urban traffic network. The result is also effectively verified the validity of the proposed models.
\end{abstract}

Keywords: urban traffic, traffic network, traffic information complexity, coordinated evolution, complexity degree.

\section{Notations}

ATC - air traffic control;

BRT - bus rapid transit;

$\mathrm{CPT}$ - conventional public transport;

CTOD - China TOD;

MRT - mass rail transit;

NBT - normal bus transit;

RBF - radial basis function;

SVM - support vector machine;

TOD - transit oriented development;

TOPSIS - technique for order of preference by similarity to ideal solution.

\section{Introduction}

Traffic system is a dynamic nonlinear and complexity system including traffic network, traffic flow, vehicle speed, congestion, and other traffic conditions. The traffic information complexity is a synthesize value helping the ordinary man intuitively know the traffic condition of a road, a street or even a city for well making their travel deci- sions and planning. Therefore, how to accurately measure the traffic information complexity has become one of the hottest topics in the academic and practical fields of transportation.

The domestic and foreign scholars have constructed some measurement models from different angles and have attempted to scientifically measure the traffic information complexity. Wang and Zheng (2005) early began studying the measurement model for the planning of urban bicycle traffic network in China. They only proposed a conception of bicycle network planning and managing through analysing urban traffic composition, but they verified that the bicycle network was an important part of urban traffic network in China. Zhao et al. (2009) introduced the concept of complex network and then proposed two approaches to extracting urban transportation systems as complex networks for solving the problem of traffic information complexity. They mainly focused on the statistical features of network evolution. In order to solve the sustainability problem of MRT, Li et al. (2010) proposed a TOD plan-

*Corresponding author. E-mail: qizhouhu@126.com 
ning model. They put forward the objective functions and constraints of CTOD model and designed the algorithm of this multi-objective optimal problem, one of which is the complexity of traffic network (MRT network). They mainly focused on the objective functions of operation costs and land-using level. Zhang and Deng (2012) established SVM comprehensive evaluation method to evaluate the development level of economic circle traffic network. They mainly focused on getting the appropriate RBF core function value of SVM for the measurement model (comprehensive evaluation method), and verifying the validity of their proposed model through comparing with Neural network method and matter-element method. Jiang (2014) proposed a new method based on multi-layer vector network for studying the efficiency of traffic planning. He first analysed several existed traffic planning systems and then pointed out the shortcomings of the methods dealing with the network nodes in a traffic network. It is important that he divided the whole network nodes into several pieces so that it is easier to calculate the complex nodes problem. In that paper, author mainly focused on the efficiency of traffic planning through the traffic network nodes. The idea of network layering in our paper comes from his approach. Wang and Li (2017) provided traffic information to all travellers is an effective method to induce each individual to make a more rational routechoice decision after they considered both the high complexity of urban traffic flow systems and the bounded rationality of travellers. However, they mainly focused on revealing the relationship between the evolution process of network traffic flow and the strategy of providing traffic information. Batishcheva and Ganichev (2018) thought human factor ranked first among causes of road accidents, and they proposed a number of organizational and technical measures to improve safety of complex road junction including use of redundant road markings.

Recently, more researches on traffic information complexity have been transferred to the air traffic fields. Krstić Simić and Babić (2015) presented the research on the relationship between airport traffic complexity and time and environmental efficiency for different ATC tactics and mainly emphasized the evaluation of airport traffic complexity, aircraft fuel consumption, gas emissions and time efficiency for different ATC tactics and/or airport airfield layouts. They proposed a measure of air traffic complexity called "dynamic complexity" for finding a tool, which could indicate potential delay generators. Aimed at increasing airspace capacity and traffic efficiency but reducing the cost while preserving safety, Radanovic et al. (2018) proposed an innovative separation management service to shift the completely centralized tactical ATC interventions to more efficient decentralized tactical operations. When they explained the relationships of air traffic factors, they further analysed the traffic complexity taking into consideration the so-called domino effect, i.e. a number of the surrounding aircraft causally involved in the separation management service by means of identification of the spatiotemporal interdependencies between them and the conflicting aircraft. That content of spatiotemporal interdependencies also inspires us when we considering the traffic network dependency.

Nevertheless, these studies still have some limitations, which can be summarized as follows: (1) the proposed measurement models are not comprehensive enough for accurately measuring the traffic information complexity (Zhang et al. 2014; Suzuki et al. 2012; Sun 2014; Chen et al. 2013; Chen 2013; Liu 2011); (2) the existing researches are mainly based on the static measured data so that the models lack dynamic factors for evaluating the complexity of multi-layer traffic network in practice (Li et al. 2013; Zhou et al. 2011; Zhu et al. 2014). In addition, their studies were mainly focus on the single-layer traffic network, and did not from the aspect of whole traffic network (Wang, Li 2017; Batishcheva, Ganichev 2018).

In order to measure the complexity of multi-layer urban traffic information more comprehensively and dynamically, in this paper, we put forward a new measurement model for dealing with above problems from the angle of data complexity. In the meantime, the information entropy theory is usually used for quantizing substance, information and attribute. Therefore, we use the multidimensional connection numbers of the information entropy theory to establish an entropy model for measuring the traffic information complexity. As defining the ordered parameters of the complexity, the coordinated evolution model of multi-layer traffic network is established.

The rest of the paper is organized as follows: Section 1 introduces the concept of traffic complexity, and then gives the definitions and formulas of traffic information complexity using the information entropy theory. In Section 2, we firstly describe the coordinated evolution mechanism of the multi-layer traffic network, and establish 4 models of the single-layer networks respectively, and propose the order degree criterions of the ordered parameters. In Section 3, we propose the coordinated evolution model for the multi-layer traffic network based on the previous proposed models. In Section 4, we verify the effectiveness and the feasibility of the proposed models through the case of Hangzhou. Conclusions are available in the last section.

\section{Traffic network complexity}

Urban traffic network is the component of whole traffic system. Therefore, a reasonable planning usually plays an important role in the urban road-network for facilitating the urban life and improving the life quality, and solving other urban traffic problems (Hu, Sun 2013).

The traffic information complexity is a kind of data complexity, which can reflect the static and dynamic aspects of traffic information when the traffic system is running (Zeng, Li 2011; Yu et al. 2013). In addition, the traffic information complexity is composed of different measurement indexes with a multi-dimensional measurement domain, and each measurement index has different influences on the data complexity. Therefore, it is necessary to adopt a new method for solving the traffic complexity problem. The multi-dimensional connection theory as a 
mathematical method is usually used for studying the uncertainty theory, and the core concept of the uncertainty theory is treating the objective thing as a systematic dimension that using the deterministic measurement or uncertainty measurement (Liu et al. 2015). On the basis of above analysis, we can study the complexity problem with the multi-dimensional connection numbers, and establish a coordinated evolution measurement model for the multi-layer traffic network.

\subsection{Equations of traffic information complexity}

\section{Definition 1. Traffic information complexity}

Assuming at time $t$, there are $\mathrm{n}$ measurement indexes $i_{1}$, $i_{2}, i_{n}$ to measure the information complexity of the traffic network, and each measurement index should meet the preferences and independent conditions. We regard the $n$ measurements as a $n$-dimensional vector with $\overrightarrow{i_{1}}, \vec{i}, \vec{i}$, and then use the utility function to express the traffic information complexity:

$$
\mu(t)=c_{1} \cdot \vec{i}_{1}+c_{2} \cdot \vec{i}_{2}+\ldots+c_{n} \cdot \vec{i}_{n},
$$

where: $\vec{i}_{1}, \vec{i}_{2}, \ldots, \vec{i}_{n}$ represent $n$ measurement indexes respectively; $c_{1}, c_{2}, \ldots, c_{n} \in R$ are the dimensional coefficients of the corresponding measurement indexes $\vec{i}_{1}, \vec{i}_{2}$, ..., $\vec{i}_{n}$, which can be obtained through the quantitative calculation or qualitative analysis. The specific calculating process of the dimensional coefficient is showed in the references (Ferreira, Couto 2013; Codina et al. 2014). Hereby, $t$ is referred to each traffic network $N W_{i}$.

\section{Definition 2. Calculation formula of traffic information complexity}

Assuming the value range of the measurement objective $X$ is $\left\{x_{1}, x_{2}, \ldots, x_{n}\right\}$, and the average information contributed rate of each measurement factor corresponding to the overall complexity is $f\left(x_{i}\right)=\frac{n_{i}}{n}$, we introduce the information entropy function (Zhu et al. 2014) to describe the traffic information complexity of $X$ :

$$
E(x)=\sum_{i=1}^{n} f(x) \cdot \ln f(x)=\sum_{i=1}^{n} \frac{n_{i}}{n} \cdot \ln \frac{n_{i}}{n},
$$

where: $E(x)$ represents the information content of the traffic information complexity; $n_{i}$ represents the traffic information content of the $i$-th single-layer traffic network; $n$ represents the whole traffic information content of the multi-layer traffic network.

We can measure the traffic information complexity using the Equation (2), and also use the multi-dimensional connection number theory to establish the measurement model of traffic information complexity from two aspects of the static and dynamic information.

Thus, there are 6 sub-dimensions information entropy models for the traffic information complexity. Furthermore, we will introduce the form of Equations (1) and (2) to construct the traffic information complexity equation in next sections.

\subsection{Complexity of different traffic networks}

Traffic information is complicate, and it is difficulty to choose the correct parameters. If choosing less parameters of the traffic information, the ordinated evolution process of the multi-layer traffic network cannot be reflected availably. Otherwise, choosing more parameters could cause the difficulties in modelling and generate the repeated information. Therefore, we choose the parameters from the views of the space and the time, which is mainly according to the traffic characteristics. From the view of space, we choose three parameters (the macro, the medium and the micro information). From the view of time, we choose two parameters (the static information and the dynamic information)

Urban traffic network is usually divided into 4-layers: bicycle traffic network $N W_{1}$, conventional public traffic network $N W_{2}$, rapid public transport (BRT transportation network) network $N W_{3}$ and rail transit network $N W_{4}$. This paper defines the performance parameters of traffic network from the traffic characteristics after analyse various form of traffic network, which are showed in Table 1.

According to Table 1, the information entropy model for urban traffic network can be constructed by using the form of Equation (1):

$$
N W_{i}(I)=c_{i 1} \cdot \vec{i}_{1}+c_{i 2} \cdot \vec{i}_{2}+c_{i 3} \cdot \vec{i}_{3}+c_{i 4} \cdot \vec{i}_{4}+c_{i 5} \cdot \vec{i}_{5},
$$

where: $N W_{i}(I)$ represents the $i$-th single-layer traffic network, $i=1,2,3,4$ respectively represent 4 -layers of the whole urban traffic networks (bicycle traffic network, conventional public traffic network, rapid public traffic network and rail transit network); $I=\left[\vec{i}_{1}, \vec{i}_{2}, \ldots, \vec{i}_{5}\right]$ is the measurement index of the performance parameters of the multi-layer urban traffic network.

\section{Information entropy model of traffic network compactness $c_{i 1}$}

Usually the "compactness" is an effective way to express the information complexity distinction between two traffic networks, so that we think of using the information entropy to measure the traffic information complexity.

Table 1. Basic parameters of each traffic network

\begin{tabular}{|l|c|c|c|c|c|}
\hline \multirow{2}{*}{ Name } & \multicolumn{5}{|c|}{ Basic parameter } \\
\cline { 2 - 6 } & Compactness & Relevancy & Connectivity & Node degree & Average degree \\
\hline \multirow{2}{*}{$\begin{array}{l}\text { Traffic network } N W_{i}, \\
i=1,2,3,4\end{array}$} & $c_{i 1}$ & $c_{i 2}$ & $c_{i 3}$ & $c_{i 4}$ & $c_{i 5}$ \\
\cline { 2 - 6 } & macro information & static information & dynamic information & micro information & medium information \\
\hline
\end{tabular}


The information entropy model for traffic network compactness can be constructed by using the form of Equation (2):

$$
c_{i 1}=-\frac{n_{i 11}}{n_{i 1}} \cdot \ln \frac{n_{i 11}}{n_{i 1}}
$$

where: $c_{i 1}$ represents the compactness of traffic networks; $n_{i 1}$ represents the defined "macro information" containing all the traffic information (including the construction information of traffic road, feature, etc.) at the $i$-th singlelayer traffic network; $n_{i 11}$ represents the "macro information" that containing the $i$-th kind of the measurement parameter at that network layer.

Hereby, $n_{i 1}$ is not the same parameter to $n$ in Equation (2), and $n_{i 11}$ is not the same parameter to $n_{i}$ in Equation (2).

\section{Information entropy model of relevancy degree $c_{i 2}$ between different traffic networks}

Originally, the relevancy degree represents the relationship between two things. Actually, in mathematics, it can be refer to express the similarity degree between two functions. Similarly, the information entropy model for the relevancy between traffic networks can be constructed using the form of Equation (2):

$$
c_{i 2}=-\frac{n_{i 21}}{n_{i 2}} \cdot \ln \frac{n_{i 21}}{n_{i 2}},
$$

where: $c_{i 2}$ represents the relevancy degree; $n_{i 2}$ represents the defined static traffic information at the $i$-th layer traffic network (including some basic parameters of traffic roads, length, width, etc.); $n_{i 21}$ represents the static traffic information of the $i$-th measurement parameter.

Hereby, $n_{i 2}$ is not the same parameter to $n$ in Equation (2), and $n_{i 21}$ is not the same parameter to $n_{i}$ in Equation (2).

\section{Information entropy model of traffic network connectivity $c_{i 3}$}

In the traffic network, arbitrary pair of the network nodes, which keeps at least one node connecting to another node, that is called the "connectivity". The information entropy model for traffic network connectivity is constructed using the form of Equation (2):

$$
c_{i 3}=-\frac{n_{i 31}}{n_{i 3}} \cdot \ln \frac{n_{i 31}}{n_{i 3}},
$$

where: $c_{i 3}$ represents the connectivity between the traffic networks; $n_{i 3}$ represents the dynamic information that containing all the information of the $i$-th single-layer traffic network (the number of the nodes); $n_{i 31}$ represents the dynamic information that containing the $i$-th kind of the measurement parameters at that network layer.

Hereby, $n_{i 3}$ is not the same parameter to $n$ in Equation (2), and $n_{i 31}$ is not the same parameter to $n_{i}$ in Equation (2).

\section{Information entropy model of traffic network node degree $c_{i 4}$}

In traffic network, the node degree is the number of edges that associating the nodes with each other. The information entropy model of traffic network of node degree is constructed using the form of Equation (2):

$$
c_{i 4}=-\frac{n_{i 41}}{n_{i 4}} \cdot \ln \frac{n_{i 41}}{n_{i 4}},
$$

where: $c_{i 4}$ represents the network node degree; $n_{i 4}$ represents the micro traffic information containing the $i$-th layer traffic network; $n_{i 41}$ represents the micro traffic information containing the $i$-th measurement parameter at that network layer.

Hereby, $n_{i 4}$ is not the same parameter to $n$ in Equation (2), and $n_{i 41}$ is not the same parameter to $n_{i}$ in Equation (2).

\section{Information entropy model of average degree of traffic network $c_{i 5}$}

Traffic information structure can determine the spatial dimension of traffic information database, while the time dimension of database is determined by the size of traffic information. In addition, the number of data in traffic information has the most directly affect data complexity. Therefore, the traffic information complexity can be calculated from the tuple number of traffic information database. The information entropy model for average degree of traffic network is constructed using the form of Equation (2):

$$
c_{i 5}=-\frac{n_{i 51}}{n_{i 5}} \cdot \ln \frac{n_{i 51}}{n_{i 5}}
$$

where: $c_{i 5}$ represents the average degree of the $i$-th singlelayer traffic network; $n_{i 5}$ represents the medium traffic information, which is containing the information of the $i$-th single-layer traffic network; $n_{i 51}$ represents the medium traffic information that containing the $i$-th measurement parameter at that network layer.

Hereby, $n_{i 5}$ is not the same parameter to $n$ in Equation (2), and $n_{i 51}$ is not the same parameter to $n_{i}$ in Equation (2).

\section{Traffic network complexity}

We first comprehensively consider the development situation of the urban traffic network, in particular, analyse the coordinated evolution process of the multi-layer urban traffic network. Relative to the single-layer traffic network, the multi-layer traffic network is more complex and is hard to express its complexity degree.

\subsection{Complexity of different traffic networks}

The purpose of constructing the coordinated evolution model of multi-layer urban traffic network is to transform the topological structure of independent urban traffic 
Table 2. Coordinated evolution index of traffic network

\begin{tabular}{|l|c|c|c|c|c|c|}
\hline \multicolumn{2}{|c|}{ Name } & Compactness & Relevancy & Connectivity & Node degree & Average degree \\
\hline Bicycle traffic network & $N W_{1}$ & $c_{11}$ & $c_{12}$ & $c_{13}$ & $c_{14}$ & $c_{15}$ \\
\hline CPT network & $N W_{2}$ & $c_{21}$ & $c_{22}$ & $c_{23}$ & $c_{24}$ & $c_{25}$ \\
\hline BRT traffic network & $N W_{3}$ & $c_{31}$ & $c_{32}$ & $c_{33}$ & $c_{34}$ & $c_{35}$ \\
\hline Rail traffic network & $N W_{4}$ & $c_{41}$ & $c_{42}$ & $c_{43}$ & $c_{44}$ & $c_{45}$ \\
\hline
\end{tabular}

network and clearly express the complexity degree of the multi-layer urban traffic network. According to analyse the characteristics of the complex network (multi-layer network), different objectives: bicycle traffic network, conventional public traffic network, BRT and the rail transit network are evolved coordinately for integrating the indexes of the multi-layer traffic network.

Table 2 gives the specific coordinated evolution index about the multi-layer traffic network using the proposed concept of Section 1.2, in particular, the Table 1. Here, we use the multi-dimensional connection number to construct the coordinated evolution model of the 4-layers network for analysing the coordinated evolution process of traffic network, which are showed as follow.

\section{Evolution model of bicycle traffic network}

In urban traffic network, the bicycle network is usually based on the existing urban road networks, which is composed by many bicycle networks. The bicycle traffic network is essential to urban transportation, so that it is considered as one layer of multi-layer traffic network. The evolution model of bicycle traffic network can be constructed referring to the form of Equation (3):

$$
\mu_{1}=c_{11} \cdot \vec{i}_{1}+c_{12} \cdot \vec{i}_{2}+c_{13} \cdot \vec{i}_{3}+c_{14} \cdot \vec{i}_{4}+c_{15} \cdot \vec{i}_{5} .
$$

\section{Evolution model of BRT network}

The urban BRT system is a new-style public passenger transport system that is between the RRT and the NBT. The BRT uses the modern bus technology and matches up the intelligent transportation technology and the operation management to open the bus routes, and to set up new-style bus stations, so it can realize new rail transitmode operation services. Now the BRT system is very common in the urban traffic network. Thus, the BRT is considered as one layer of the multi-layer traffic network. The evolution model for BRT can be constructed referring to the form of Equation (3):

$$
\mu_{2}=c_{21} \cdot \vec{i}_{1}+c_{22} \cdot \vec{i}_{2}+c_{23} \cdot \vec{i}_{3}+c_{24} \cdot \vec{i}_{4}+c_{25} \cdot \vec{i}_{5} .
$$

\section{Evolution model of CPT network}

The CPT is the most common traffic network, which refers to the vehicles for passenger travelling. The CPT owns the fixed route with a regular time. It is also considered as one layer of the multi-layer traffic network. The evolution model for conventional public traffic network can be constructed referring to the form of Equation (3):

$$
\mu_{3}=c_{31} \cdot \vec{i}_{1}+c_{32} \cdot \vec{i}_{2}+c_{33} \cdot \vec{i}_{3}+c_{34} \cdot \vec{i}_{4}+c_{35} \cdot \vec{i}_{5} .
$$

\section{Evolution model of rail transit network}

The rail transit is a kind of transportations having the features of large volume, fast speed, safety, punctuality, environment protection, energy saving and land using. The subway, light railway, fast railway, tram and other traffic modes are belonged to the categories of rail transit. The evolution model of rail transit network can be constructed as the form of Equation (3):

$$
\mu_{4}=c_{41} \cdot \vec{i}_{1}+c_{42} \cdot \vec{i}_{2}+c_{43} \cdot \vec{i}_{3}+c_{44} \cdot \vec{i}_{4}+c_{45} \cdot \vec{i}_{5} .
$$

\subsection{Order degree model of multi-layer traffic network}

Because of the complexity and uncertainty of the urban transportation system, it is impossible to distinguish the change degree of the ordered parameters over time, or to find the accurate ordered parameters with coordinate transformation. Nevertheless, the concert theory regards the order degree as the evolution direction of one system, and it can determine the system's coordinated order state. Therefore, the ordered parameters can only be estimated by the criterion of ordered parameters. In addition, the ordered parameters existing the upper and lower threshold ranges, which represent the ideal and the non-ideal states of the parameters, and we can set the threshold ranges refer to the experience from developed countries and Chinese urban traffic development. Therefore, we decide to introduce the concept of order degree to measure the coordination of the multi-layer traffic network.

As the concert theory showed, the ordered parameters from each layer of the traffic network should stay in a certain range of critical threshold that ensuring the multi-layer traffic network system to evolve coordinately and orderly. That means the range should be greater than the minimum critical value, and lesser than the maximum critical value.

Supposing the $j$-th ordered parameter (here $j=1,2$, $3,4,5)$ is $c_{i j}$ when it is at the $i$-th layer of the multi-layer traffic network (here $i=1,2,3,4,5$ ), the minimum critical threshold is $c_{i j \max }$ and the maximum critical threshold is $c_{i j \max }$. Obeying the above definition, $c_{i j}$ should stay in the critical threshold range, which meets $c_{i j} \in\left[c_{i j \min }, c_{i j \max }\right]$. Based on the critical threshold values above, the estimation criterions about the order degrees of the ordered parameters, and of the single-layer and the multi-layer traffic network are built as following sections. 


\subsubsection{Criterion about the order degree} of the traffic network ordered parameters

The traffic network ordered parameters can be divided into three types according to the properties of multi-attribute data (Brito, De Almeida 2012): Those are cost type, benefit type and fixed type. Obeying the commensurability of different parameters, the criterion about the order degree of traffic network ordered parameters can be constructed by the quantization values.

\section{Estimation criteria for the benefit-type ordered parameter}

Supposing the ordered parameter value $c_{i j}$ under the threshold interval is bigger, and the order degree of the $i$-th single-layer traffic network is higher, otherwise, the order degree is lower. Such parameter is called the benefittype ordered parameter. The order degree $u_{i j}$ of the $j$-th ordered parameter of the $i$-th single-layer traffic network can be calculated by the equation:

$$
u_{i j}\left(c_{i j}\right)=\frac{c_{i j}-c_{i j \min }}{c_{i j \max }-c_{i j \min }} .
$$

\section{Estimation criteria for the cost-type ordered parameter}

Similarly, supposing the ordered parameter value $c_{i j}$ under the threshold interval is smaller, and the order degree of the $i$-th single-layer traffic network is lower. Otherwise, $c_{i j}$ is bigger, the order degree of the $i$-th single-layer is higher. Such parameter is called the cost-type ordered parameter. Thus, the order degree $u_{i j}$ of the $j$-th ordered parameter of the $i$-th single-layer traffic network can be calculated by the equation:

$$
u_{i j}\left(c_{i j}\right)=\frac{c_{i j \max }-c_{i j}}{c_{i j \max }-c_{i j \min }} .
$$

\section{Estimation criteria for the fixed-type ordered parameter}

If the ordered parameter $c_{i j}$ is closer to a certain value $c$ in the range of threshold interval, the order degree of the $i$-th layer traffic network is higher. Otherwise, $c_{i j}$ is far away from $c$, and the order degree of the $i$-th single-layer traffic network is lower. Such parameter is called the fixed-type ordered parameter. The order degree $u_{i j}$ of the $j$-th ordered parameters of the $i$-th single-layer traffic network is:

$$
u_{i j}\left(c_{i j}\right)=1-\frac{\left|c_{i j}-c\right|}{c_{i j \max }-c_{i j \min }} .
$$

It is necessary to estimate the correct type according to the characteristics of ordered parameters firstly and to choose the corresponding calculated formula. Referring to the ordered parameters of Table 2 and the references at home and abroad (Lindau et al. 2014; Hassannayebi et al. 2014; Ferreira, Couto 2013; Codina et al. 2014), this paper gives out the variable range of the order degree and of the ordered parameters (including the parameter type) of the 4-layers traffic network, which are showed in Table 3.

\subsubsection{Estimation criterion for the order degree of the single-layer traffic network}

Through calculating the order degree of the single-layer traffic network to analyse the characteristics of the singlelayer traffic network in different periods, and then revealing the coordinated evolution mechanism among the multi-layer traffic networks (conventional traffic network, BRT traffic network, rail traffic network, bicycle traffic network, etc.). Therefore, the order degree $u_{i j}\left(c_{i j}\right)$ can be used as a feedback factor of the $i$-th layer traffic network for the evolution process of the multi-layer traffic network. If meeting $u_{i j}\left(c_{i j}\right) \in[0,1]$, the $i$-th layer traffic network evolves toward to the order direction, it states the setting plan of this layer traffic network is feasible. After estimating all the values of $u_{i j}\left(c_{i j}\right)$ in the $i$-th layer traffic network, the order degree of the $i$-th single-layer traffic network is:

$$
r_{i}=\sum_{j=1}^{5} \omega_{i j} \cdot u_{i j}\left(c_{i j}\right),
$$

where: $r_{i}$ is the order degree of the $i$-th single-layer traffic network, $i=1,2,3,4 ; \omega_{i j}$ is the weight coefficient of the ordered parameter $x_{i j}, \omega_{i j} \geq 0, \sum_{j=1}^{5} \omega_{i j}=1$.

If the order degree of the $i$-th layer traffic network is greater, which indicates the coordinated degree of this layer traffic network is higher and the system function is

Table 3. Threshold interval of the ordered parameters and the order degree of the 4-layer traffic network

\begin{tabular}{|l|c|c|c|c|c|}
\hline \multirow{2}{*}{ Name } & \multicolumn{5}{|c|}{ Threshold range } \\
\cline { 2 - 6 } & Compactness & Relevancy & Connectivity & Node degree & Average degree \\
\cline { 2 - 6 } & Benefit type & Benefit type & Benefit type & Cost type & Fixed type \\
\hline $\begin{array}{l}\text { Order of the order parameter of } \\
\text { bicycle traffic network }\end{array}$ & {$[0.3,0.5]$} & {$[0.7,0.9]$} & {$[0.7,0.9]$} & {$[0.5,0.7]$} & 0.5 \\
\hline Order of the order parameter of CPT & {$[0.7,0.9]$} & {$[0.6,0.8]$} & {$[0.7,0.9]$} & {$[0.3,0.5]$} & 0.7 \\
\hline $\begin{array}{l}\text { Order of the order parameter of BRT } \\
\text { traffic network }\end{array}$ & {$[0.2,0.4]$} & {$[0.3,0.5]$} & {$[0.2,0.4]$} & {$[0.2,0.4]$} & 0.3 \\
\hline $\begin{array}{l}\text { Order of the order parameter of rail } \\
\text { traffic network }\end{array}$ & {$[0.1,0.3]$} & {$[0.2,0.4]$} & {$[0.1,0.3]$} & {$[0.1,0.3]$} & 0.1 \\
\hline
\end{tabular}


more efficient. Otherwise, the system function is less efficient. That is the estimation criterion for the order degree of the single-layer traffic network.

\subsubsection{Estimation criterion for the order degree of the multi-layer traffic network}

For the urban, its traffic system is composed of the multilayer traffic network, and the multi-layer traffic network is composed of many single-layer traffic networks. The urban traffic network, which is defined to be composed of the 4-layer traffic network, the single-layer networks are the conventional traffic network, the BRT network, the rail transit network and the bicycle traffic network respectively. Thus, this paper constructs the order degree function of the multi-layer urban traffic network from the 4 single-layer traffic networks:

$$
R=\sum_{i=1}^{4} \omega_{i} \cdot r_{i},
$$

where: $R$ is the order degree of the multi-layer urban traffic network; $\omega_{i}$ is the weight coefficient of $r_{i}\left(r_{i}\right.$ is the order degree of the $i$-th single-layer traffic network), $\omega_{i} \geq 0$, $\sum_{i=1}^{4} \omega_{i}=1$.

If the order degree $R$ is greater (approaching to 1 ), which means the coordinated degree of the multi-layer urban traffic network is higher, and its system function is more efficient. Otherwise, $R$ is smaller (approaching to 0 ), the coordinated degree is lower, and it indicates the function of this urban traffic system is less efficient.

\section{Coordinated evolution model of the multi-layer urban traffic network}

From the view of the concert theory, the coordinated evolution model of the multi-layer urban traffic network can reflect the complementary competition and the coordinated evolution mechanism of the multi-layer traffic network. This paper establishes the coordinated evolution model by the proposed order degree model.

\subsection{Hypothesis of the traffic network coordinated evolution}

For the multi-layer traffic network (setting the 4-layers traffic network as the research object), supposing that (see below).

The ordered parameter set of the traffic network $I=\left(i_{1}, i_{2}, i_{3}, i_{4}, i_{5}\right)$ equals to the set $\{$ compactness, relevancy, connectivity, node degree, average degree $\}$. The traffic network set $N W=\left(N W_{1}, N W_{2}, N W_{3}, N W_{4}\right)$ equals to the set \{bicycle traffic network, CPT network, BRT, rail transit network\}. The weight vector of the ordered parameters of the $i$-th single-layer traffic network $\omega_{i}=\left(\omega_{i 1}, \omega_{i 2}, \omega_{i 3}, \omega_{i 4}, \omega_{i 5}\right)$, and the set meets $\omega_{i j} \geq 0$, $\sum_{j=1}^{5} \omega_{i j}=1$
Using the order degree of the single-layer traffic networks to measure the coordinated degree of 4-layer traffic network in city $\mathrm{A}$, and then constructing the coordinated evolution matrix of city A:

$$
D(A)=\left[\begin{array}{l}
c_{11} \cdot \vec{i}_{1}+c_{12} \vec{i}_{2}+c_{13} \vec{i}_{3}+c_{14} \cdot \vec{i}_{4}+c_{15} \cdot \vec{i}_{5} \\
c_{21} \cdot \vec{i}_{1}+c_{22} \cdot \vec{i}_{2}+c_{23} \cdot \vec{i}_{3}+c_{24} \cdot \vec{i}_{4}+c_{25} \cdot \vec{i}_{5} \\
c_{31} \cdot \vec{i}_{1}+c_{32} \cdot \vec{i}_{2}+c_{33} \cdot \vec{i}_{3}+c_{34} \cdot \vec{i}_{4}+c_{35} \cdot \vec{i}_{5} \\
c_{41} \cdot \vec{i}_{1}+c_{42} \cdot \vec{i}_{2}+c_{43} \cdot \vec{i}_{3}+c_{44} \cdot \vec{i}_{4}+c_{45} \cdot \vec{i}_{5}
\end{array}\right] .
$$

\subsection{Coordinated evolution model for the multi-layer urban traffic network}

The coordinated evolution model is established by using the theory of the multi-dimensional connection numbers. For accurately obtaining the value of the comprehensive coordinated evolution, we design a complete calculated process of the coordinated evolution model for the multilayer urban traffic network (Figure 1).

\section{Step 1}

Determining the weight vector $W_{i}=\left(w_{i 1}, w_{i 2}, w_{i 3}, w_{i 4}, w_{i 5}\right)$ of the order degree of the $i$-th single-layer traffic network, $i=1,2,3,4$.

In order to reduce the subjectivity in the calculation process, we use Equation (19) of the fuzzy deviation weight method to calculate the weight coefficient of $I$ :

$$
w_{i j}=\Delta_{i j} \cdot\left(\sum_{i=1}^{5} \Delta_{i j}\right)^{-1} \text {, }
$$

where: $\Delta_{i j}=\frac{1}{4} \cdot \sum_{i=1}^{4}\left|b_{i j}-\bar{b}_{j}\right|, \bar{b}_{j}=\frac{1}{4} \cdot \sum_{i=1}^{4} b_{i j}, b_{i j}$ is the ordered parameter value.

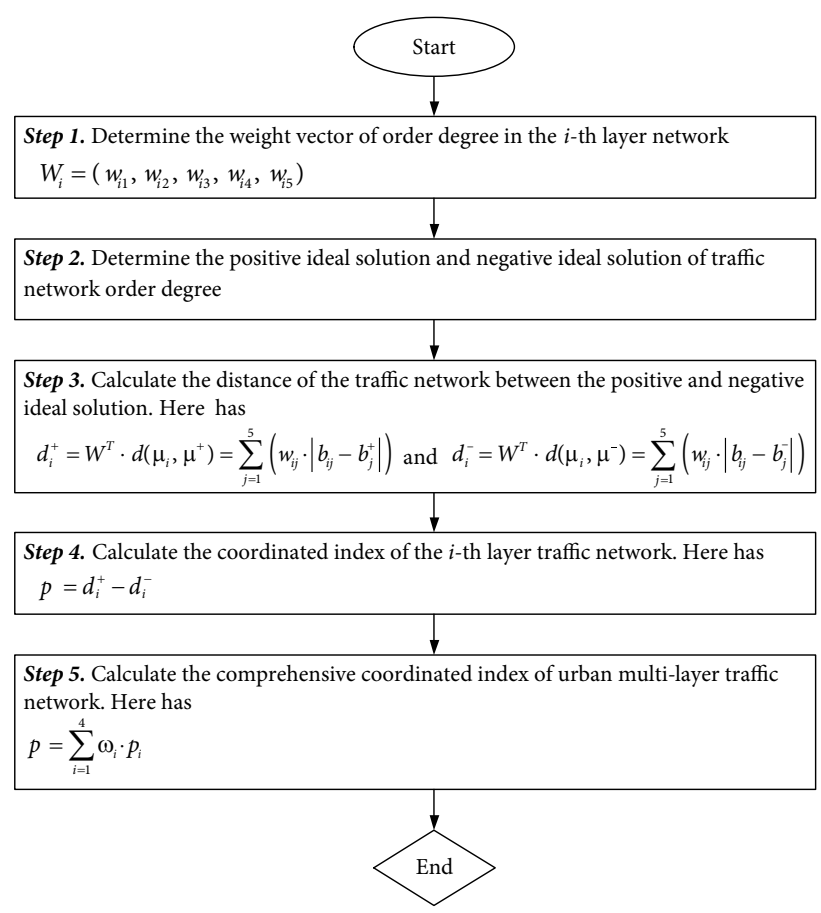

Figure 1. The calculated process of the coordinated evolution model 


\section{Step 2}

Determining the positive ideal solution and the negative ideal solution of the order degree. The concepts of the positive ideal solution and the negative ideal solution are from the TOPSIS theory (Zhu et al. 2014), it is usually used the "distance" to express the difference degree between different values. The purpose is to construct these solutions (those are also called the optimal solution and the worst solution) for the ordered parameter indexes.

Setting $c_{j}^{+}=\max _{1 \leq i \leq 4} c_{i j}$ is the maximum critical threshold, $c_{j}^{-}=\min _{1 \leq i \leq 4} c_{i j}(j=1,2, \ldots, 5)$ is the minimum critical threshold, the positive ideal solution is $\mu^{+}=c_{1}^{+} \cdot \vec{i}_{1}+$ $c_{2}^{+} \cdot \vec{i}_{2}+c_{3}^{+} \cdot \vec{i}_{3}+c_{4}^{+} \cdot \vec{i}_{4}+c_{5}^{+} \cdot \vec{i}_{5}$, and the negative ideal solution is $\mu^{-}=c_{1}^{-} \cdot \vec{i}_{1}+c_{2}^{-} \cdot \vec{i}_{2}+c_{3}^{-} \cdot \vec{i}_{3}+c_{4}^{-} \cdot \vec{i}_{4}+c_{5}^{-} \cdot \vec{i}_{5}$.

\section{Step 3}

Calculating the distance between the positive solution and the negative ideal solution. Setting the equation of the positive distance is: $d\left(\mu_{i}, \mu^{+}\right)=\sum_{j=1}^{5}\left|b_{i j}-b_{j}^{+}\right|$, the distance from the $i$-th layer traffic network to the positive ideal solution can be described as Equation (20) (Shih et al. 2007; Zhu et al. 2014):

$$
d_{i}^{+}=W^{T} \cdot d\left(\mu_{i}, \mu^{+}\right)=\sum_{j=1}^{5}\left(w_{i j} \cdot\left|b_{i j}-b_{j}^{+}\right|\right) .
$$

Similarly, setting the equation of the negative distance is: $d\left(\mu_{i}, \mu^{-}\right)=\sum_{j=1}^{5}\left|b_{i j}-b_{j}^{-}\right|$, the distance from the $i$-th layer traffic network to the negative ideal solution can be described as Equation (21):

\section{Step 4}

$$
d_{i}^{-}=W^{T} \cdot d\left(\mu_{i}, \mu^{-}\right)=\sum_{j=1}^{5}\left(w_{i j} \cdot\left|b_{i j}-b_{j}^{-}\right|\right) .
$$

Calculating the coordinated index of the $i$-th layer traffic network. According to the distance from the $i$-th layer traffic network to the absolute positive ideal solution and the negative ideal solution, the coordinated index of the $i$-th single-layer traffic network is:

$$
p_{i}=d_{i}^{+}-d_{i}^{-},
$$

where: $p_{i}$ is the coordinated index of the $i$-th single-layer traffic network, $i=1,2,3,4$.

\section{Step 5}

Calculating the comprehensive coordinated index of urban multi-layer traffic network, set:

$$
p=\sum_{i=1}^{4} \omega_{i} \cdot p_{i},
$$

where: $p$ is the comprehensive coordinated index of urban multi-layer traffic network; $\omega_{i}$ is the weight coefficient of the coordinated index $p_{i}$ in the $i$-th layer traffic network, $\omega_{i} \geq 0, \sum_{i=1}^{4} \omega_{i}=1$.
The purpose of calculating the comprehensive coordinated index of the multi-layer traffic network is to quantize the ordinated development degree of the multi-layer traffic network, then to reflect the coordinated evolution degree and the operation quality. It gives the city manager and the researcher the reference for solving the uncertainty, randomness and fuzziness problems of the city. The index value is in the range of $[0,0.5]$, the larger the better. The quality of the traffic network can be preferred through sort $p$. Hereby, $p$ is greater that indicates the coordinated evolution process of this transportation is better.

\section{Case analysis}

An example of the coordinated evolution of the multi-layer traffic network is analysed by Hangzhou urban traffic network in China, and it is mainly analysed for verifying the feasibility and effectiveness of the proposed methods with the specific calculation process. After our research team (High-Speed Railway Science Research Institute of Nanjing University of Science and Technology, China) surveyed and refer to the official transportation report 2017 of Hangzhou, the traffic network of Hangzhou is consisted of 4 sub-networks, such as the bicycle traffic network, the BRT network, the rail transit network and the conventional traffic network. Figure 2 shows the routes of 4 traffic networks in Hangzhou we surveyed before, and they can be combined to a whole traffic network of Hangzhou. In addition, the purpose of dividing the multi-layer network into 4 single-layer network is to analyse and calculate the comprehensive degree of the complex network conveniently and intuitively.

The survey data include the required characteristic data (5 basic parameters we mentioned in Section 1.2) of each traffic network mentioned in Table 1, specific having road length, width, quantity, traffic flow, service stations and so on. According to the expert analysis extensive investigation and the standard range of threshold interval for each basic parameters given in Table 3, our research team uses the information entropy models from Equations (4)-(8) and the coordinated evolution models of the single-layer traffic networks from Equations (9)-(12) with the urban traffic parameters, and to build a coordinated evolution matrix $D(A)$ of the 4 single-layer traffic networks of Hangzhou, the matrix of quantized values are showed as follow:

$$
D(A)=\left[\begin{array}{l}
0.41 \cdot \vec{i}_{1}+0.82 \cdot \vec{i}_{2}+0.83 \cdot \vec{i}_{3}+0.65 \cdot \vec{i}_{4}+0.45 \cdot \vec{i}_{5} \\
0.78 \cdot \vec{i}_{1}+0.73 \cdot \vec{i}_{2}+0.75 \cdot \vec{i}_{3}+0.43 \cdot \vec{i}_{4}+0.61 \cdot \vec{i}_{5} \\
0.33 \cdot \vec{i}_{1}+0.41 \cdot \vec{i}_{2}+0.37 \cdot \vec{i}_{3}+0.29 \cdot \vec{i}_{4}+0.23 \cdot \vec{i}_{5} \\
0.22 \cdot \vec{i}_{1}+0.29 \cdot \vec{i}_{2}+0.18 \cdot \vec{i}_{3}+0.21 \cdot \vec{i}_{4}+0.17 \cdot \vec{i}_{5}
\end{array}\right],
$$

where: $D(A)$ can basically represents the relationships between the whole traffic network and 4 sub-networks. 
a)

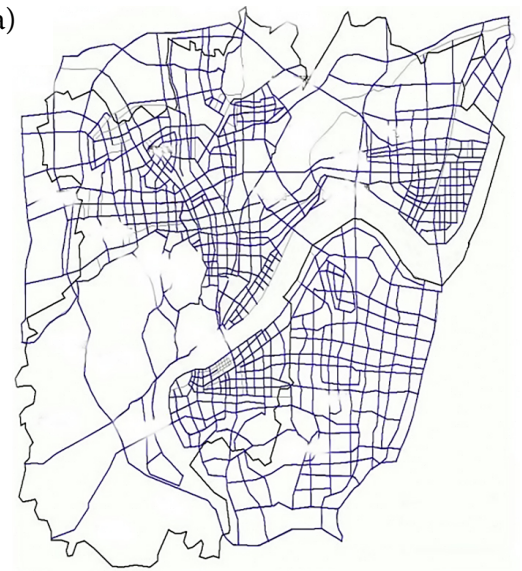

c)

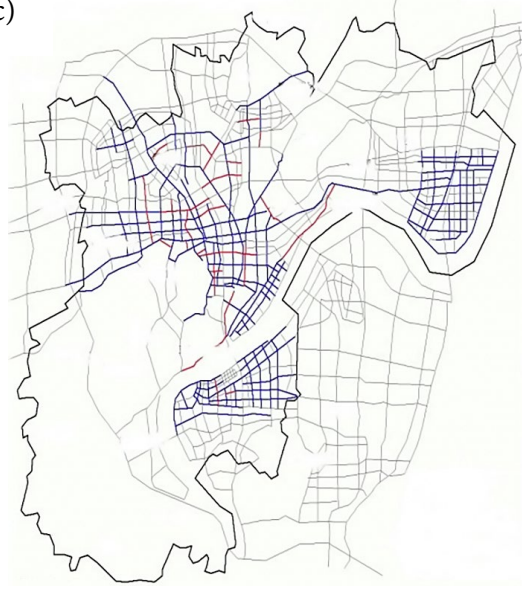

b)

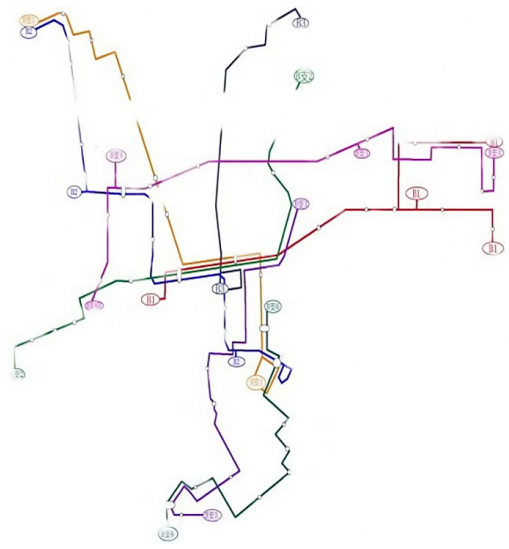

d)

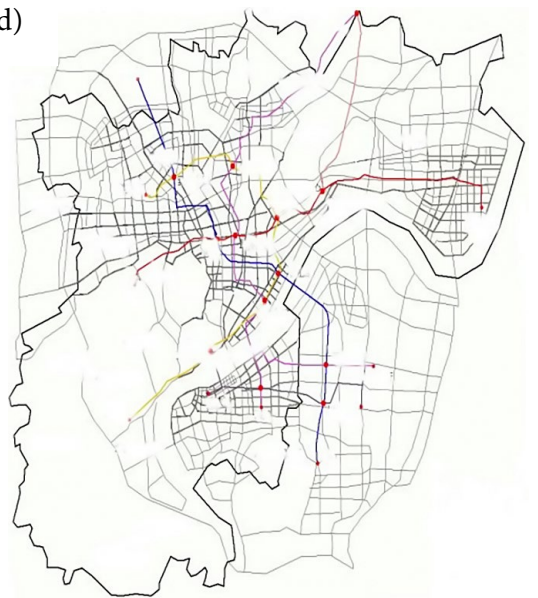

Figure 2. Multi-layer traffic network in Hangzhou: a - bicycle traffic network; b - BRT network; $\mathrm{c}$ - rail transit network; $\mathrm{d}$ - conventional traffic network

\subsection{The order degree of the ordinated evolution model for the traffic network of Hangzhou}

According to Equations (16) and (17), the order degree $r_{1}$ of the bicycle traffic network $N W_{1}$ is 0.7812 . Similarly, the order degree $r_{2}$ of the conventional traffic network $N W_{2}$ is 0.6753 , the order degree $r_{3}$ of the BRT network $N W_{3}$ is 0.8739 , and the order degree $r_{4}$ of the rail transit network $N W_{4}$ is 0.7358 . Then using Equations (17)-(19) to calculate the order degree $R$ of the multi-layer traffic network (whole traffic network) of Hangzhou, and the value is 0.7929 . Hereby, $R$ is approaching to 1 , which means the coordinated degree of the multi-layer traffic network is relatively high, and the quality of the traffic system of Hangzhou is high.

\subsection{The coordinated evolution model for the multi-layer traffic network of Hangzhou}

The calculation process of the coordinated evolution model of the multi-layer traffic network in Hangzhou is calculated as the steps in Section 3:

\section{Step 1}

Determining the weight coefficient vector of the order degree of the Hangzhou traffic network. The weight co- efficient vectors of the 4-kinds of the single-layer traffic networks are showed as described below.

As Figure 3 showed, the weight vector of the bicycle traffic network is:

$$
W_{1}=(0.1831,0.2026,0.2053,0.1939,0.1949) \text {, }
$$

the weight vector of conventional traffic network is:

$$
W_{2}=(0.2135,0.1763,0.2517,0.1678,0.1707),
$$

the weight vector of BRT network is:

$$
W_{3}=(0.2005,0.1697,0.2713,0.1757,0.1628),
$$

and the weight vector of rail transit traffic network is:

$$
W_{4}=(0.1935,0.1903,0.2377,0.1767,0.1819),
$$

where: $W_{1}, W_{2}, W_{3}, W_{4}$ are the weight vectors of each single-layer traffic network (There are 4 sub-networks: $\left.N W_{1} \ldots N W_{4}\right)$; and there are 5 weight coefficients $-w_{i 1}$, $w_{i 2}, w_{i 3}, w_{i 4}, w_{i 5}$ - in each sub-network has to correspond to the 5 basic parameters.

Step 2

Determining the positive ideal and the negative ideal solution of the order degree of the single-layer traffic network. According the TOPOSIS theory (Shih et al. 2007), we can 


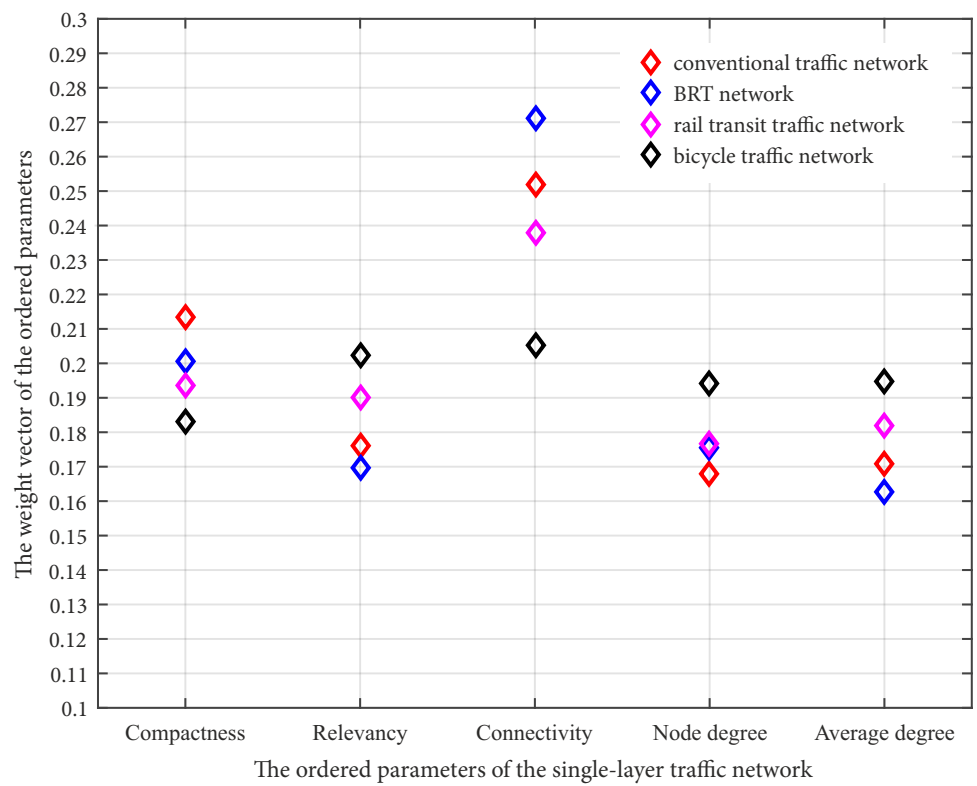

Figure 3. Weight vectors of the 4 kinds of the single-layer traffic networks in Hangzhou

first get the order evaluation values with each sub-network, and the method is showed in Equation (29):

$$
\left[\begin{array}{ccc}
f_{11} & \cdots & f_{1 j} \\
\vdots & \ddots & \vdots \\
f_{i 1} & \cdots & f_{i j}
\end{array}\right]=\left[\begin{array}{ccc}
c_{11} & \cdots & c_{1 j} \\
\vdots & \ddots & 0 \\
c_{i 1} & \cdots & c_{i j}
\end{array}\right] \cdot\left[\begin{array}{ccc}
W_{1} & 0 & 0 \\
0 & \ddots & 0 \\
0 & 0 & W_{j}
\end{array}\right] .
$$

Then calculating the positive ideal solution: $f_{i}^{+}=\max \left(f_{i j}\right)$, when $c_{i j}$ is belonged to benefit type or $f_{i}^{+}=\min \left(f_{i j}\right)$, when $c_{i j}$ is belonged to cost type. In addition, calculating the negative ideal solution is: $f_{i}^{-}=\min \left(f_{i j}\right)$, when $c_{i j}$ is belonged to benefit type or $f_{i}^{-}=\max \left(f_{i j}\right)$, when $c_{i j}$ is belonged to cost type. All types of $c_{i j}$ have been given in Table 3 .

Hereby, the positive ideal solution of each single-layer traffic network is:

$$
\mu^{+}=0.9 \cdot \overline{i_{1}}+0.9 \cdot \overline{i_{2}}+0.9 \cdot \overline{i_{3}}+0.7 \cdot \bar{i}_{4}+0.7 \cdot \bar{i}_{5},
$$

and the negative ideal solution one is:

$$
\mu^{-}=0.1 \cdot \bar{i}_{1}+0.2 \cdot \bar{i}_{2}+0.1 \cdot \bar{i}_{3}+0.1 \cdot \bar{i}_{4}+0.1 \cdot \bar{i}_{5} .
$$

\section{Step 3}

Calculating the distance of the traffic network between the positive and negative ideal solution. The distance of the positive ideal solution of the multi-layer traffic network in Hangzhou is $d_{i}^{+}=1.2309$, the distance of the negative ideal solution is $d_{i}^{-}=0.9037$ after calculating by Equations (20) and (21).

\section{Step 4}

Calculating the traffic network coordinated index in Hangzhou. The coordinated index values of the 4 single-

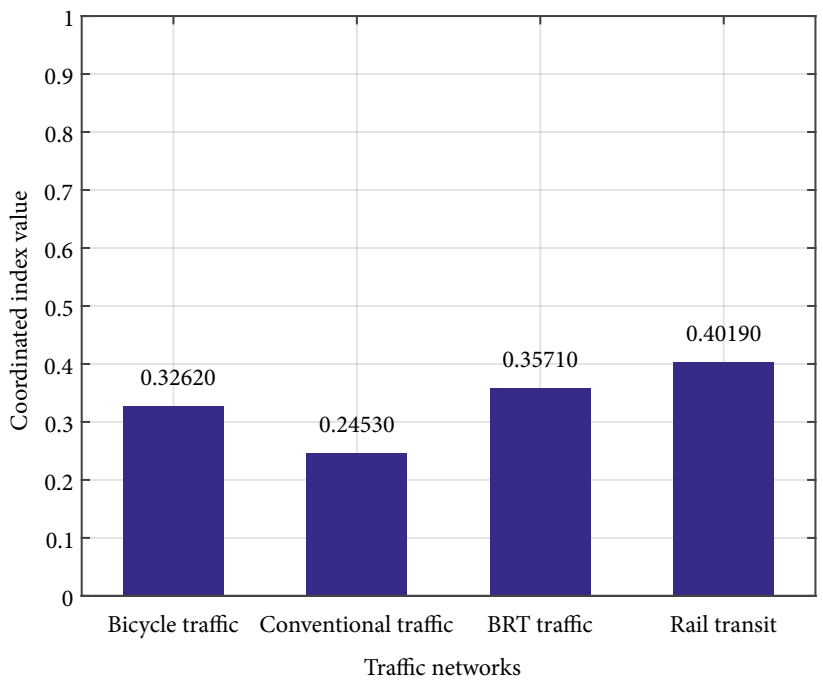

Figure 4. The coordinated index of traffic network in Hangzhou

layer traffic networks in Hangzhou are calculated from Equation (22) are showed in Figure 4.

$p$ is the coordinated evolution index of each traffic sub-network, which is calculated from Equations (20)(23) based on the coordinated evolution model. There are 2 kinds of $p$ in this model. The first $p$ is $p_{i}$, which is the coordinated index of the $i$-th single-layer traffic network. And it can be calculated by Equations (20)-(22). The second $p$ is the coordinated evolution index of the whole traffic network, which can be calculated by Equation (23) after we obtain the 4 weight vectors of $W_{1}, W_{2}, W_{3}, W_{4}$. As the calculated result, the coordinated index of the bicycle traffic network $p_{1}=0.3262$. The coordinated index of BRT traffic network $p_{2}=0.3571$, the coordinated index of conventional traffic network $p_{3}=0.2453$, and the coordinated index of rail transit network $p_{4}=0.4019$. 


\section{Step 5}

Calculating the comprehensive coordinated index of the multi-layer traffic network. According to the calculation means of Equation (23), the comprehensive coordinated index $p$ of multi-layer traffic network of Hangzhou is 0.3323 . As $p=0.3323$ of the multi-layer traffic network is between 0 and 0.5 , the degree of the coordinated evolution of Hangzhou is relatively higher, which reaches the expected value, $p$ is also consistent with the estimation criterion of Section 2.2.3. Furthermore, according to the official transportation report 2017 from Hangzhou hall, it shows the traffic congestion ranking of Hangzhou is from the 45 to the 57-th (relatively high) in China, the utilization of main urban traffic network (rail transit network) is $40.6 \%$ (it almost meets $p_{4}=0.4019$ ), and the general public transport satisfaction has reached $91.3 \%$. Thus, the proposed coordinated evolution model for the multi-layer traffic network is consistent with the actual development trend in Hangzhou, and is also consistent with the results of the transportation report.

\section{Conclusions}

Accurately evaluate the ordinated evolution degree of the multi-layer urban traffic network is a complex problem. The main difficulty includes the multi-layer network division and the modelling of the single-layer network. This paper constructs a measurement model of the multi-layer traffic network based on the information complexity. It starts from the view of single-layer network for establishing the 5-dimensions (compactness, relevancy, connectivity, node degree, average degree) information entropy model of the ordered parameters of each single-layer network. As to the information entropy theory, the information entropy model can cover the weakness of the complex information quantization, especially the extension of the traffic information in the dimension and width. After that, this paper constructs the ordered parameters degree model of the multi-layer traffic network by the 4 order degrees of these single-layer networks. Finally, the coordinated evolution model is established based on the ordered parameters degree of the multi-layer urban traffic network. The real case analysis shows the proposed model is valid.

The proposed model can calculate the traffic information complexity, and reduce the characteristic parameters of the multi-layer network. It also provides a theoretical foundation for calculating the quantized value of the multi-layer urban traffic, which can intuitively evaluate the ordinated development degree of this city. Furthermore, the model provides the basic and quantitative guides for solving the coordinated evolution problems of multi-layer traffic network and other fields.

\section{Acknowledgements}

The work was supported in part by Grant National Natural Science Foundation of China (Grant No 51178157), high-level project of the top six talents in Jiangsu province
(Grant No JXQC-021), the Key science and technology program in Henan province (Grant No 182102310004), and the Humanities and Social science research Programs Foundation of Ministry of Education of China (Grant No 18YJAZH028).

\section{Author contributions}

The authors confirm contribution to the paper as follows:

- Qizhou Hu: manuscript preparation, study conception and design, manuscript writing;

- Minjia Tan: data collection, literature search and review, model analysis and manuscript revision.

\section{Disclosure statement}

No potential conflict of interest was reported by the authors.

\section{References}

Batishcheva, O.; Ganichev, A. 2018. Measures to improve traffic safety at road junctions, Transportation Research Procedia 36: 37-43. https://doi.org/10.1016/j.trpro.2018.12.040

Brito, A. J.; De Almeida, A. T. 2012. Modeling a multi-attribute utility newsvendor with partial backlogging, European Journal of Operational Research 220(3): 820-830.

https://doi.org/10.1016/j.ejor.2012.02.027

Chen, C. 2013. Road public transportation planning based on rail transit network in Shanghai, Urban Mass Transit 16(9): 87-90. (in Chinese).

https://doi.org/10.3969/j.issn.1007-869X.2013.09.021

Chen, S.; Liu, D.; Jiang, Y. 2013. The optimization methodology of bus network around city-metro corridors based on the key performance indicator analysis, Modern Urban Research (1): 23-28. (in Chinese).

https://doi.org/10.3969/j.issn.1009-6000.2013.01.007

Codina, E.; Marín, Á.; Cadarso, L. 2014. Robust infrastructure design in rapid transit rail systems, Transportation Research Procedia 3: 660-669. https://doi.org/10.1016/j.trpro.2014.10.045

Ferreira, S.; Couto, A. 2013. Urban road network safety model at the transportation planning process, Journal of Transportation Safety \& Security 5(1): 46-65.

https://doi.org/10.1080/19439962.2012.710300

Hassannayebi, E.; Sajedinejad, A.; Mardani, S. 2014. Urban rail transit planning using a two-stage simulation-based optimization approach, Simulation Modelling Practice and Theory 49: 151-166. https://doi.org/10.1016/j.simpat.2014.09.004

Hu, Q.-Z.; Sun, X. 2013. Model for traffic congestion state monitor in urban road network based on multi-dimension connection number, China Journal of Highway and Transport 26(6): 143-149. (in Chinese).

https://doi.org/10.3969/j.issn.1001-7372.2013.06.020

Jiang, G.-X. 2014. High efficient traffic planning based on guidance of multi-vector network, Computer Simulation 31(4): 192-195. (in Chinese). https://doi.org/10.3969/j.issn.1006-9348.2014.04.044

Krstić Simić, T.; Babić, O. 2015. Airport traffic complexity and environment efficiency metrics for evaluation of ATM measures, Journal of Air Transport Management 42: 260-271. https://doi.org/10.1016/j.jairtraman.2014.11.008 
Li, Y.; Guo, H. L.; Li, H.; Xu, G. H.; Wang, Z. R.; Kong, C. W. 2010. Transit-oriented land planning model considering sustainability of mass rail transit, Journal of Urban Planning and Development 136(3): 243-248. https://doi.org/10.1061/(ASCE)0733-9488(2010)136:3(243)

Li, Z.; Chen, X.; Ji, S. 2013. Transit network optimization method based on BRT attracting region - a case study of Changzhou, Journal of Wuhan University of Technology (Transportation Science \& Engineering) 37(3): 491-495. (in Chinese). https://doi.org/10.3963/j.issn.2095-3844.2013.03.010

Lindau, L. A.; Hidalgo, D.; De Almeida Lobo, A. 2014. Barriers to planning and implementing bus rapid transit systems, $R e$ search in Transportation Economics 48: 9-15. https://doi.org/10.1016/j.retrec.2014.09.026

Liu, W.-B. 2011. Road public traffic network optimization model based on rail transit network, Journal of Xihua University (Natural Science Edition) 30(1): 12-15. (in Chinese). https://doi.org/10.3969/j.issn.1673-159X.2011.01.004

Liu, Y.; Zhu, N.; Ma, S.-F. 2015. Simultaneous optimization of transit network and public bicycle station network, Journal of Central South University 22(4): 1574-1584. https://doi.org/10.1007/s11771-015-2674-8

Radanovic, M.; Piera-Eroles, M. A.; Koca, T.; Ramos González, J. J. 2018. Surrounding traffic complexity analysis for efficient and stable conflict resolution, Transportation Research Part C: Emerging Technologies 95: 105-124. https://doi.org/10.1016/j.trc.2018.07.017

Shih, H.-S.; Shyur, H.-J.; Lee, E. S. 2007. An extension of TOPSIS for group decision making, Mathematical and Computer Modelling 45(7-8): 801-813.

https://doi.org/10.1016/j.mcm.2006.03.023

Sun, F.-P. 2014. Comprehensive evaluation model of multi-step construction of urban rail transit, Transportation Standardization (14): 11-13. (in Chinese).

https://doi.org//10.16503/j.cnki.2095-9931.2014.14.004

Suzuki, K.; Kanda, U.; Doi, K.; Tsuchizaki, N. 2012. Proposal and application of a new method for bicycle network planning, Procedia - Social and Behavioral Sciences 43: 558-570. https://doi.org/10.1016/j.sbspro.2012.04.129

Wang, J.; Li, S. 2017. Modeling and simulation of network traffic flow evolution based on incomplete information feedback strategies in the ATIS environment, International Journal of Modeling, Simulation, and Scientific Computing 8(3): 1750031. https://doi.org/10.1142/S1793962317500313

Wang, Q.-P.; Zheng, A.-L. 2005. Tentative plan for building city cycle-traffic network, Urban Problems (6): 85-89. (in Chinese). https://doi.org/10.3969/j.issn.1002-2031.2005.06.019

Yu, X.-X.; Li, Y.; Lu, H.-P. 2013. A model for discrete traffic network design based on real option, Journal of Highway and Transportation Research and Development 30(7): 126-132. (in Chinese). https://doi.org/10.3969/j.issn.1002-0268.2013.07.021

Zeng, M.-H.; Li, X.-M. 2011. Optimal allocation approach of transport network resource based on hierarchical property, Journal of Central South University 42(1): 247-253. (in Chinese).

Zhang, B.; Deng, W. 2012. SVM evaluation method of transportation network in economic circle, Journal of Southeast University (Natural Science Edition) 42(6): 1227-1232. (in Chinese). https://doi.org/10.3969/j.issn.1001-0505.2012.06.037

Zhang, K.; Qin, B.-B.; Liu, Y.-S.; Zhang, Q. 2014. Research on the evaluation of urban rail transit network, Journal of Railway Engineering Society (3): 97-101. (in Chinese). https://doi.org/10.3969/j.issn.1006-2106.2014.03.018
Zhao, Y.; Du, W.; Chen, S. 2009. Application of complex network theory to urban transportation network analysis, Urban Transport of China 7(1): 57-65. (in Chinese). https://doi.org/10.3969/j.issn.1672-5328.2009.01.017

Zhou, Y.; Deng, W.; Hu, Q. 2011. Study on the optimization of public transit network based on genetic algorithm and tabu search algorithm, Journal of Wuhan University of Technology (Transportation Science \& Engineering) 35(1): 42-45. (in Chinese). https://doi.org/10.3963/j.issn.1006-2823.2011.01.010

Zhu, W.-T.; Bian, Z.-Y.; Li, H.-P. 2014. The optimization scheme of public traffic network based on information entropy and TOPSIS, Journal of Xihua University (Natural Science Edition) (5): 94-97. (in Chinese).

https://doi.org/10.3969/j.issn.1673-159X.2014.05.021 